\title{
Performed Study Agent Transfer
}

National Cancer Institute

\section{Source}

National Cancer Institute. Performed Study Agent Transfer. NCI Thesaurus. Code C93437.

The completed action in which an authorized party at a designated study site dispenses or receives a study agent to/from a study subject. 